\title{
SINGULAR SPECTRUM ANALYSIS FOR HETEROGENEOUS TIME SERIES BY TAKING ITS FORMAL ERRORS INTO ACCOUNT
}

\author{
Fengwei WANG ${ }^{1)}$, Yunzhong SHEN ${ }^{1) *}$, Weiwei LI ${ }^{2)}$ and Qiujie CHEN ${ }^{1)}$ \\ 1) College of Surveying and Geo-informatics, Tongji University, Shanghai, PR, China \\ ${ }^{2)}$ College of Geomatics, Shandong University of Science and Technology, Qingdao, PR, China
}

*Corresponding author's e-mail: yzshen@tongji.edu.cn

\begin{tabular}{l} 
ARTICLE INFO \\
\hline Article history: \\
Received 23 September 2018 \\
Accepted 4 December 2018 \\
Available online 12 December 2018 \\
\hline
\end{tabular}

Keywords:

Time series analysis

Singular spectrum analysis

Stabilizing weights

Formal error

\begin{abstract}
Since the formal errors of many geodetic time series are also available, this paper proposes a new singular spectrum analysis (SSA) approach with stabilizing weights (SSAW) by taking the formal errors into account, where the weight of time series data is constructed based on the ratio of formal error to signal power spectrum. The formulae of the proposed SSAW are derived in detail and then used to process the real Global Mean Sea Level (GMSL) time series compared to the traditional SSA. When the first 10 principal components are used to fit the GMSL time series, the fitting errors of the SSAW and traditional SSA are $4.80 \mathrm{~mm}$ and $5.14 \mathrm{~mm}$, with the reduction of $6.61 \%$. According to the 500 simulations based on the reconstructed signals and formal errors of GMSL time series, the mean root mean squared errors and mean absolute errors of reconstructed signals using the SSAW relative to traditional SSA are reduced from $2.18 \mathrm{~mm}$ to $1.66 \mathrm{~mm}$ and $1.67 \mathrm{~mm}$ to $1.34 \mathrm{~mm}$, respectively. Therefore, if the formal errors of a noisy time series given, the proposed SSAW approach is suggested to analyze this time series.
\end{abstract}

\section{INTRODUCTION}

Singular Spectrum Analysis (SSA) has been successfully applied to extract the trend and periodic signals from a time series without prior knowledge (Broomhead and King, 1986; Vautard and Ghil, 1989; Golyandina et al., 2001). In SSA a trajectory matrix is first constructed from a time series, and then principal component analysis is carried out to pick out dominant components. The signals of the time series are reconstructed with the dominant components and the remaining components are regarded as the noise (Sivapragasam et al., 2001; Vitanov et al., 2008). SSA has been widely used in geosciences, e.g. the stream flow and sea-surface temperature analysis (Robertson and Mechoso, 1998; Kondrashov and Ghil, 2006; Chen and Sneeuw, 2016; Zotov et al., 2017), the seismic tomography (Oropeza and Sacchi, 2011; Tiwari and Rajesh, 2014; Rajesh and Tiwari, 2014), earth rotation and climate variability (Zotov et al., 2016), the gravity anomaly separation (Kumar et al., 2017) and monthly gravity field analysis (Zotov and Shum, 2010; Rangelova et al., 2012). For processing an incomplete time series, several SSA approaches, such as spatiotemporal data filling (Kondrashov and Ghil, 2006), iterative data filling (Kondrashov et al., 2010), or compensation via a scale (Schoellhamer, 2001) and its improved version (Shen et al., 2015), which is based on the principle that a time series can be reconstructed with its principal components (Shen et al., 2014).
SSA can be directly used to process the time series contaminated by additive noise (Allen and Smith, 1997; Wu and Chau, 2011). When a time series is contaminated by multiplicative noise, the traditional strategy first converts the multiplicative noise to additive noise by taking log-transform (Golyandina et al., 2001), then processes it with traditional SSA. SSA outperforms other methods in extracting signals from heterogeneous time series, such as the Fourier transform, wavelet transform, empirical mode decomposition and Kalman filters (Kumar et al., 2017; Barrios-Muriel et al., 2016; Watson, 2016; Chen et al., 2016). The covariance or formal errors of a time series are usually not being considered in most SSA approaches, however, formal errors are available for many geodetic time series data. Some SSA approaches have been developed by prewhitening a time series data using its noise matrix (Cadzow, 1988) or rank-deficient covariance matrix (Hansen et al., 2005). Nevertheless, the prewhitening processing will change the structure of original time series. In order to keep the signal structure unchanged the weighted singular value decomposition (Gillard and Zhigljavsky, 2016) and weighted hankelization (Zvonarev and Golyandina, 2017) applied the weights to the trajectory matrix rows and columns, which are thereby named as weighted SSA approach. However since signals are unknown, an iterative algorithm is needed. To avoid iteration, a new SSA approach with stabilizing weights (SSAW) is developed in this 
contribution, in which the weight is constructed based on the ratio of formal error to signal power spectrum.

The rest of this paper is organized as follows: the SSAW approach is introduced after traditional SSA briefly presented in Section 2 . The sea level change signals are extracted from Global Mean Sea Level (GMSL) time series and analyzed in Section 3. Then, synthetic examples are carried out to demonstrate the performance of the SSAW compared to traditional SSA in extracting signals in Section 4, and conclusions are given in Section 5.

\section{METHODOLOGY}

\subsection{SINGULAR SPECTRUM ANALYSIS}

From a time series $x_{i}(1 \leq i \leq N)$, we can construct an $L \times K \cdot(K=N-L+1)$ trajectory matrix $\boldsymbol{X}$ with a window size $L$ (Vautard et al., 1992) as follows,

$$
\boldsymbol{X}=\left[\begin{array}{cccccc}
x_{1} & x_{2} & \cdots & x_{i+1} & \cdots & x_{K} \\
x_{2} & x_{3} & \cdots & x_{i+2} & \cdots & x_{K+1} \\
\vdots & \vdots & \vdots & \vdots & \vdots & \vdots \\
x_{L} & x_{L+1} & \cdots & x_{i+L} & \cdots & x_{N}
\end{array}\right]
$$

Its lagged covariance matrix $\boldsymbol{C}$ is formulated as $\boldsymbol{C}=\boldsymbol{X} \boldsymbol{X}^{\mathrm{T}}$. Then the matrix $\boldsymbol{C}$ is decomposed as,

$\boldsymbol{C}=\boldsymbol{V} \boldsymbol{\Lambda} \boldsymbol{V}^{T}$

where $\boldsymbol{\Lambda}$ is a diagonal matrix with eigenvalues sorted in descending order, its $k$ th number is denoted as $\lambda_{k}(1 \leq k \leq L) . \boldsymbol{V}$ is an orthogonal matrix; $\boldsymbol{v}_{k}$ is its $k$ th eigenvector. The principal component matrix $\boldsymbol{A}$ is determined by,

$$
\boldsymbol{A}=\boldsymbol{V} \boldsymbol{X}
$$

The $k$ th row vector $\boldsymbol{a}_{k}$ of $\boldsymbol{A}$ is called the $k$ th Principle Components (PCs). Then the $i$ th element $a_{k, i}$ of $\boldsymbol{a}_{k}$ is computed with,

$a_{k, i}=\sum_{j=1}^{L} x_{i+j-1} v_{k, j}, \quad 1 \leq i \leq N-L+1$

where $v_{k, j}$ is the $j$ th element of $\boldsymbol{v}_{k}$. Then the Reconstructed Components (RCs) of the time series with the $k$ th PC are represented as (Vautard et al., 1992),

$$
x_{i}^{k}= \begin{cases}\frac{1}{i} \sum_{j=1}^{i} a_{k, i-j+1} v_{k, j} & 1 \leq i \leq L-1 \\ \frac{1}{L} \sum_{j=1}^{L} a_{k, i-j+1} v_{k, j} & L \leq i \leq N-L+1 \\ \frac{1}{N-i+1} \sum_{j=i-N+L}^{L} a_{l, i-j+1} v_{k, j} & N-L+2 \leq i \leq N\end{cases}
$$

where $x_{i}^{k}$ is the $i$ th element reconstructed from $k$ th PC. Since the eigenvalues of covariance matrix $\boldsymbol{C}$ are sorted in descending order, the signal of the time series can be represented by the first several RCs, while the remaining $\mathrm{RCs}$ being treated as noise. Thus the signals of the time series are the sum of dominant $\mathrm{RCs}$ as follows,

$\hat{x}_{i}=\sum_{k=1}^{n} x_{i}^{k}, i=1,2, \cdots, N$

where $\hat{x}_{i}$ denotes the $i$ th element of reconstructed signals from the first $n$ dominant RCs.

\subsection{SINGULAR SPECTRUM ANALYSIS WITH STABILIZING WEIGHTS}

In traditional SSA approach the Equations (1) (6) are normally used to process a time series without considering its covariance or formal errors, which implies that the priori information of observational noise is neglected in traditional SSA approach. In order to use the priori covariance $\boldsymbol{Q}$, Hansen et al (2005) proposed one solution generating a new time series by prewhitening the original time series as,

$\boldsymbol{x}^{\prime}=\boldsymbol{x} \boldsymbol{Q}^{-1 / 2}$

where $\boldsymbol{x}=\left(x_{1}, x_{2}, \cdots, x_{n}\right)$ is a time series represented in row vector form. It is obvious that the covariance of the generated time series $\boldsymbol{x}^{\prime}$ is an identity matrix. Therefore, Equations (1) (6) can be applied to extract the signals $\hat{x}_{i}$ from $\boldsymbol{x}^{\prime}$. Then the signals of original time series are recovered as,

$\hat{\boldsymbol{x}}=\hat{\boldsymbol{x}}^{\prime} \boldsymbol{Q}^{1 / 2}$

Since not the full covariance $\boldsymbol{Q}$ but only its diagonal elements are available for most geodetic time series, the Equations (7) and (8) can be simplified as,

$x_{i}^{\prime}=x_{i} \frac{\sigma_{0}}{\sigma_{i}}$

and

$\hat{x}_{i}=\hat{x}_{i}^{\prime} \frac{\sigma_{i}}{\sigma_{0}}$

where the formal error $\sigma_{i}$ is the squared root of the $i$ th diagonal element of $\boldsymbol{Q}, \sigma_{0}$ is the formal error of unit weight which is computed with the principle of keeping the total energy of the time series unchanged (Li et al., 2015). However, the prewhitening processing in Equation (7) or (9) seriously changes the signal structure of the original time series, especially when $\sigma_{i}$ is varied significantly from epoch to epoch, since the transformation of Equation (9) implies that both the signals and noises of the time series are multiplied by a weight factor $\sigma_{0} / \sigma_{i}$. In the weighted SSA, an iterative procedure is needed (Gillard and Zhigljavsky, 2016; Zvonarev and Golyandina, 2017). For this reason, a new weight factor is derived in the following parts, which can suppress noise, but hold more signals than Hansen et al. (2005). 
A time series $x_{i}(1 \leq i \leq N)$ is composed of signal $s_{i}$ and noise $e_{i}$ as follows,

$x_{i}=s_{i}+e_{i}$

where $s_{i}$ is stationary with mostly low frequencies in the spectrum, $e_{i}$ consists of independent samples with slowly changing variances.

Supposing the signal and noise are independent, the variance of $x_{i}$ can be derived via the law of error propagation law as,

$\sigma_{x_{i}}^{2}=\sigma_{s_{i}}^{2}+\sigma_{i}^{2}$

where $\sigma_{s_{i}}^{2}$ is the variance of signal $s_{i}$. If both the $\sigma_{i}$ and $\sigma_{s_{i}}$ are available, we can convert the original time series as,

$x_{i}^{\prime}=\sigma_{s_{i}} x_{i} / \sqrt{\sigma_{s_{i}}^{2}+\sigma_{i}^{2}}=x_{i} / \sqrt{1+\sigma_{i}^{2} / \sigma_{s_{i}}^{2}}=x_{i} / \alpha_{i}$

where the weight factor $\alpha_{i}=\sqrt{1+\sigma_{i}^{2} / \sigma_{s_{i}}^{2}}, i=1,2, \cdots, N$ can suppress the impacts of noise. By the law of error propagation, we can derive from Equation (13) as,

$\sigma_{x_{i}^{\prime}}^{2}=\sigma_{s_{i}}^{2}$

Therefore, the converted time series $x_{i}^{\prime}$ can have the same variance as the original signals. With the converted time series $x_{i}^{\prime}$ or the correspondent trajectory matrix $\boldsymbol{X}^{\prime}$, one can form a lag covariance $C^{\prime}$ as,

$\boldsymbol{C}^{\prime}=\boldsymbol{X}^{\prime} \boldsymbol{X}^{\prime \mathrm{T}}$

The eigenvectors $v_{k, j}^{\prime}$ of the lag covariance $\boldsymbol{C}^{\prime}$ are then used to calculate the PCs $a_{k, i}^{\prime}$ with,

$a_{k, i}^{\prime}=\sum_{j=1}^{L} x_{i+j-1}^{\prime} v_{k, j}^{\prime}, 1 \leq i \leq N-L+1$

Then the RCs of the time series are calculated as Equation (5) by replacing the $a_{k, i}$ and $v_{k, j}$ with $a_{k, i}^{\prime}$ and $v_{k, j}^{\prime}$. With the signals $\hat{x}^{\prime}$ of the converted time series determined with Equation (6), the signals of the original time series is recovered with,

$\hat{x}_{i}=\hat{x}_{i}^{\prime} \alpha_{i}$

Since a new weight factor $\alpha_{i}=\sqrt{1+\sigma_{i}^{2} / \sigma_{s_{i}}^{2}}$, which is based on the ratio of $\sigma_{i}$ to $\sigma_{s_{i}}$, is introduced in our developed approach, we call it as SSAW. The weight factor $\alpha_{i}$ is larger than 1 and increases with respect to the ratio of $\sigma_{i}$ to $\sigma_{s_{i}}$. Hence the larger the noise is, the bigger the weight factor will be. Therefore, the impacts of heterogeneous noise are suppressed and the signal to noise ratio will increase in SSAW processing. When the signal variance is not known, it should be estimated beforehand. Especially, when the time series is stationary, the signal variances $\sigma_{s_{i}}^{2}$ of all epochs are equal, which can be estimated by using the reconstructed signals,

$\hat{\sigma}_{s}^{2}=\frac{1}{N-1} \sum_{i=1}^{N}\left(s_{i}-\bar{s}\right)^{2}$

where $s_{i}$ is the reconstructed signal at $i$ th epoch; $\bar{s}$ is the mean signals of all epochs; $N$ is the number of epochs of the time series.

\subsection{WINDOW SIZE AND RECONSTRUCTED ORDER}

The window size $L$ and number of dominant PCs are the two key factors to be determined in SSA. The window size mostly depends on the research topic and the priori information of the time series (Golyandina and Zhigljavsky, 2013). L should be large enough theoretically but not larger than $N / 2$ (Golyandina, 2010). If a time series contains a periodic component, the window size had better to be proportional to the period. If one is interested in signal as a whole, but not the separability among signal components, the window size is not a key factor (Golyandina et al., 2001). There are different approaches to determine the number of dominant PCs for reconstructing signals, such as the threshold for the sum contribution of dominant PCs (Figueiredo et al., 2011; Ma et al., 2011), the first inflection point in the logarithm of eigenvalues diagrams ( $\mathrm{Hu}$ et al., 2009), and the weighted correlations (w-correlation) (Golyandina et al., 2001). Due to its intuitive, wcorrelation matrix is widely used to detect the signal subspace (Golyandina et al., 2018). Thus the subspace of dominant PCs is determined with w-correlation matrix. The weights for computing weighted correlations are determined by,

$$
w_{i}= \begin{cases}i, & 0 \leq i<L \\ L, & L \leq i \leq K \\ N-i+1, & K<i \leq N\end{cases}
$$

From Equation (19), it is apparent that the weight is equal to the number of the element $x_{i}$ in the trajectory matrix $\boldsymbol{X}$. Define the inner product of two components $\mathrm{PC}^{i}$ and $\mathrm{PC}^{j}$ of length $N$ is,

$\left(\mathrm{PC}^{i}, \mathrm{PC}^{j}\right)_{w} \stackrel{d e f}{=} \sum_{k=1}^{N} w_{k} \mathrm{PC}_{k}^{i} \mathrm{PC}_{k}^{j}$

The weighted correlation to measure the degree of approximate separability between $\mathrm{PC}^{i}$ and $\mathrm{PC}^{j}$ is defined as follows (Golyandina et al., 2001),

$\rho_{i, j}^{(w)}\left(\mathrm{PC}^{i}, \mathrm{PC}^{j}\right) \stackrel{\text { def }}{=} \frac{\left(\mathrm{PC}^{i}, \mathrm{PC}^{j}\right)_{w}}{\left\|\mathrm{PC}^{i}\right\|_{w}\left\|\mathrm{PC}^{j}\right\|_{w}}$ 
If the weighted correlation is 0 , it means that the components $\mathrm{PC}^{i}$ and $\mathrm{PC}^{j}$ are fully separable. If the weighted correlation between two components $\mathrm{PC}^{i}$ and $\mathrm{PC}^{j}$ is larger than a certain threshold, then the two components are regarded as non-separable. Therefore, the PCs for reconstructing signals are determined based on the threshold $\varepsilon$ as,

$$
\max \left(\left|\rho_{i, j}^{(w)}\left(\mathrm{PC}^{i}, \mathrm{PC}^{j}\right)\right|\right)<\varepsilon, i=1,2, \cdots, L ; j=i+2, \cdots, L
$$

If the $i$ th component doesn't satisfy the Equation (22), then the reconstructed order is fixed at $i-1$.

\section{GLOBAL MEAN SEA LEVEL TIME SERIES ANALYSIS USING THE SSAW}

The GMSL time series presented in Figure 1 includes 1368 monthly averaged data spanning from 1900 to 2013, and the corresponding formal errors are presented in Figure 2, which are available from the web site (http://www.psmsl.org). Since the number of tide gauge stations continually increases all over the world, the formal errors significantly become smaller from 1900 to 2005. However, the formal errors become larger from 2005 to 2013, mainly for fewer records are available in these years due to the delay in the transmission of GMSL information to the PSMSL (Church and White, 2011). For GMSL time series, the trend, which contains more than $90 \%$ of the total variance, is pre-determined using least squares fit based on quadratic polynomial functional model (Fig. 1). The de-trended GMSL time series (after removing the trend) is shown in Figure 3 with blue solid line, which is then processed with traditional and SSAW and finally the trend is added back to the reconstructed signal.

The window size is chosen as $L=600$, which is equal to 50 years, less than half of the length of detrended GMSL time series through experiments comparison. The w-correlations are employed to determine the PCs for reconstructing de-trended GMSL signals, and the 10 leading PCs are determined when the threshold is chosen as 0.5 empirically. Thereby, 10 leading PCs (same as in SSA) are used to reconstruct signals in the SSAW. With the reconstructed de-trended GMSL signals from 10 leading PCs, we can estimate that $\hat{\sigma}_{s}^{2}=58.46 \mathrm{~mm}^{2}$. The converted de-trended GMSL time series by Equation (13) is shown in Figure 3 with red solid line.

The w-correlations between any pair of the 30 leading RCs are depicted in Figures 4 and 5 for traditional and SSAW, respectively. It is expected that the indicated pairs are 1-2, 3-4, 5-6, 7-8 and 9-10, so the RCs 1-10 are as signals and the RCs 11-600 are treated as noise. The noise and its frequency spectrum are presented in Figure 6, in which the noise power spectrum is about 10 , much less that of signal.

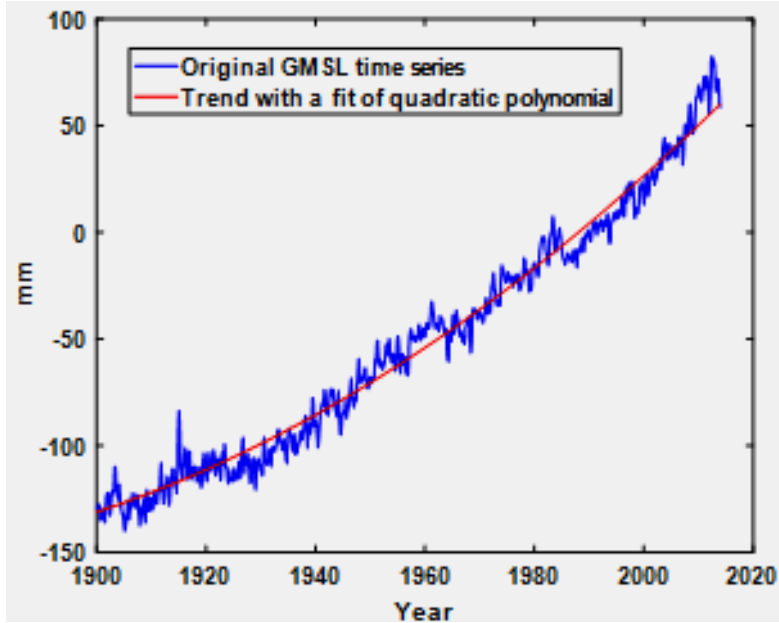

Fig. 1 Original GMSL time series and corresponding trend term.

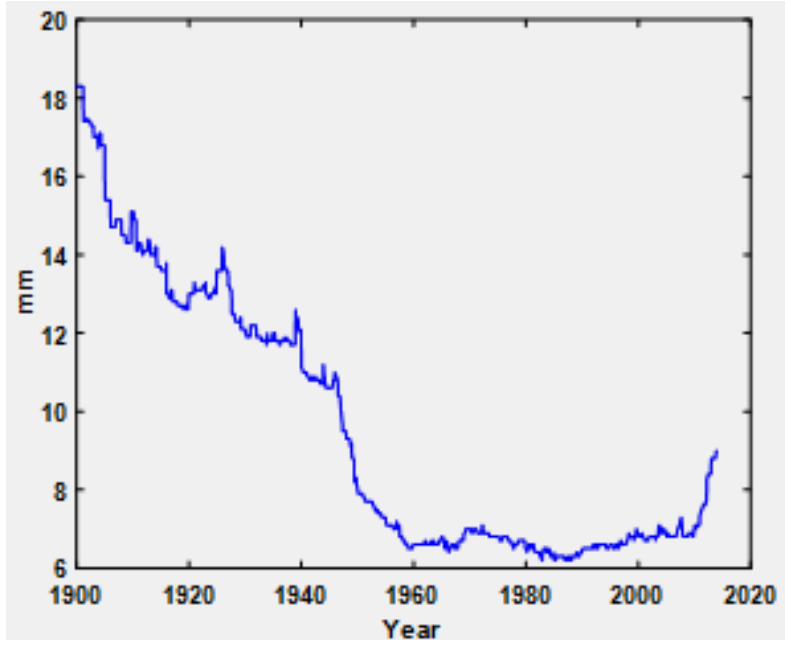

Fig. 2 Formal error series.

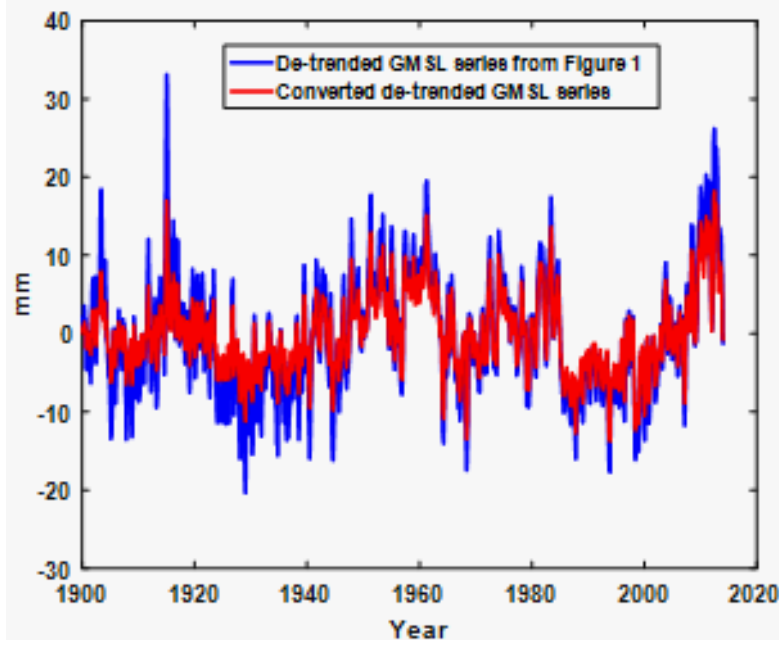

Fig. 3 The de-trended and its converted GMSL time series. 


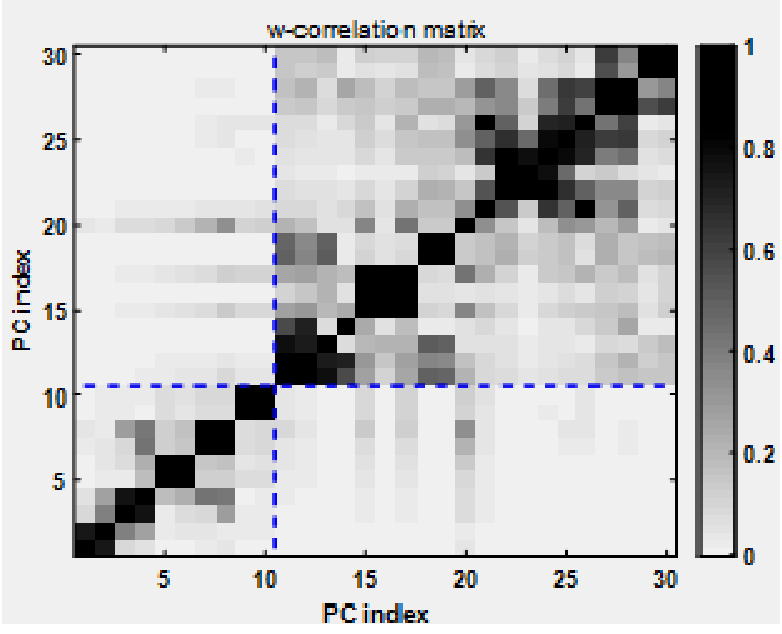

Fig. 4 The w-correlations of 30 leading RCs of traditional SSA.

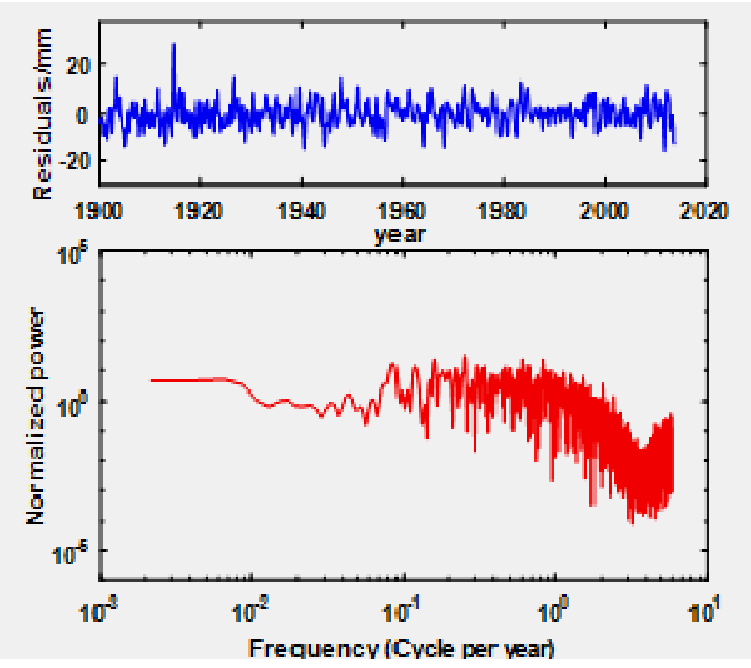

Fig. 6 The noise (RCs 11-600) of SSA (top) and its frequency spectrum (bottom).

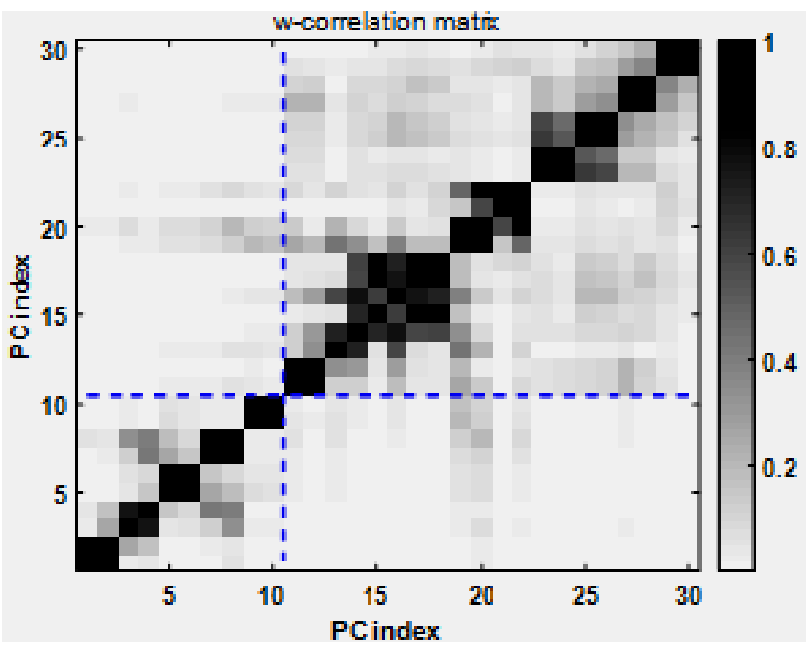

Fig. 5 The w-correlations of 30 leading RCs of SSAW.

The periodograms of the 5 indicated pairs (1-2), (3-4), (5-6), (7-8) and (9-10) are presented in Figure 7, which reveal the impacts of Pacific Decadal Oscillation (PDO), El Niño/South Oscillation (ENSO) and sunspots on the GMSL time series, since the periods of PDO, ENSO and sunspots are respectively about 20 30, 20 25 and 10 11 years (Chen et al., 1998; Nerem, 1995). The periods of the indicated pairs (3-4 and 7-8) are possibly caused by PDO and ENSO, respectively; the period of harmonic pair (5-6) is consistent with the period of sunspots. Besides, a 57-year oscillation (1-2) is significant in de-trended GMSL time series, which is in a good agreement with Church and White (2011) and Chambers et al. (2012). The periods of 5 indicated pairs of the two approaches are listed in Table 1 together with their amplitudes, which are computed with least squares fit using harmonic analysis. The results show that the differences of periods between the two approaches are very small, but differences of amplitudes are very significant.

The percentages of total variance of 10 leading PCs are shown in Figure 8 for the converted and original GMSL time series, where the PCs of the converted time series contain more energy than that of the original GMSL time series. Specially, the variance of 10 leading PCs of the converted time series is

Table 1 Periods and mean amplitudes of paired harmonic components.

\begin{tabular}{ccccc}
\hline \multirow{2}{*}{ PC index } & \multicolumn{2}{c}{ Traditional SSA } & \multicolumn{2}{c}{ SSAW } \\
\cline { 2 - 5 } & Period/year & Amplitude/mm & Period/year & Amplitude/mm \\
\hline $1-2$ & 57.00 & 4.89 & 57.00 & 5.19 \\
$3-4$ & 30.40 & 2.72 & 30.40 & 2.40 \\
$5-6$ & 10.36 & 2.10 & 10.36 & 2.29 \\
$7-8$ & 19.00 & 1.59 & 19.00 & 1.97 \\
$9-10$ & 7.12 & 1.17 & 7.24 & 1.25 \\
\hline
\end{tabular}



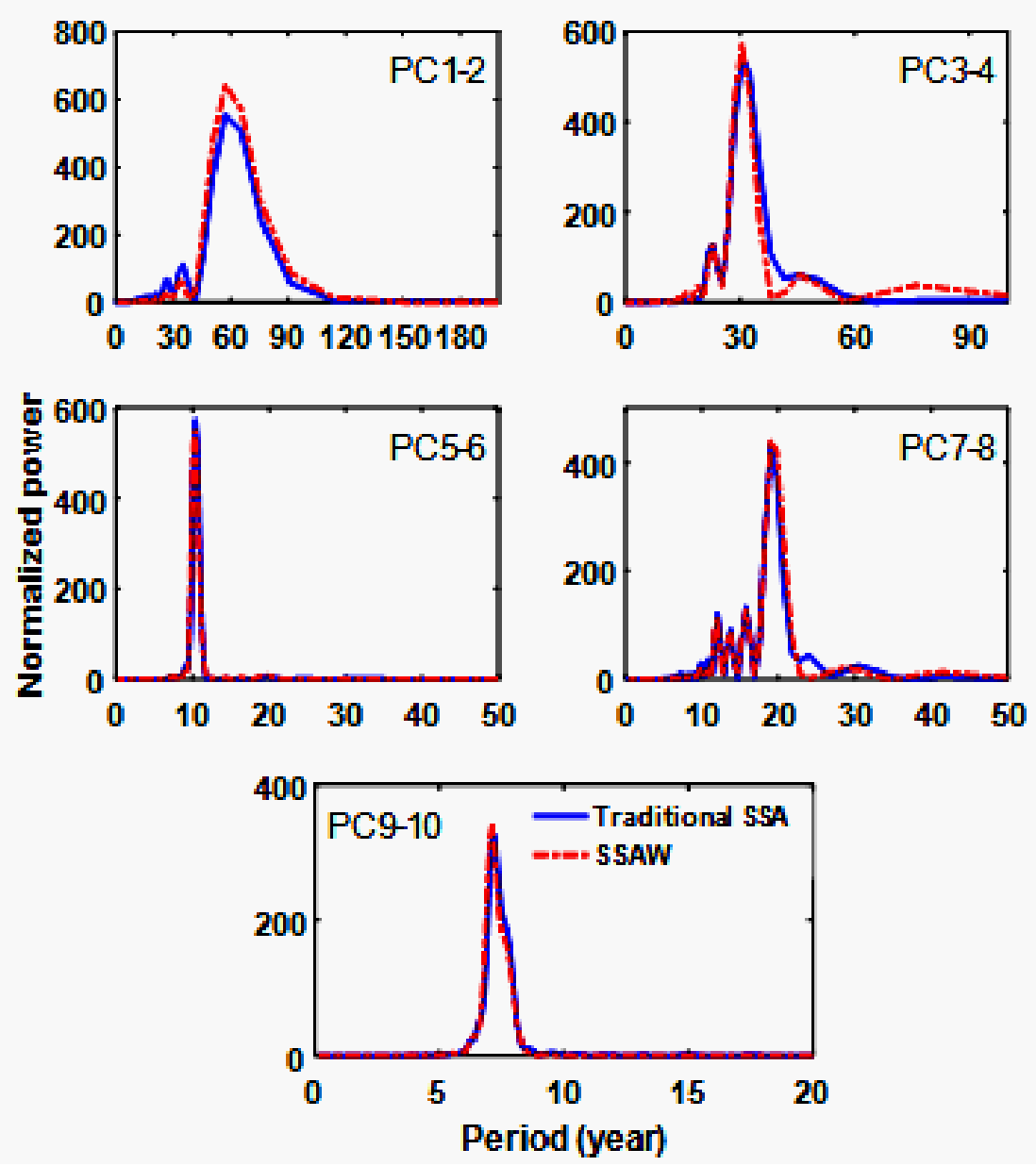

Fig. 7 The corresponding period of the paired harmonic components.

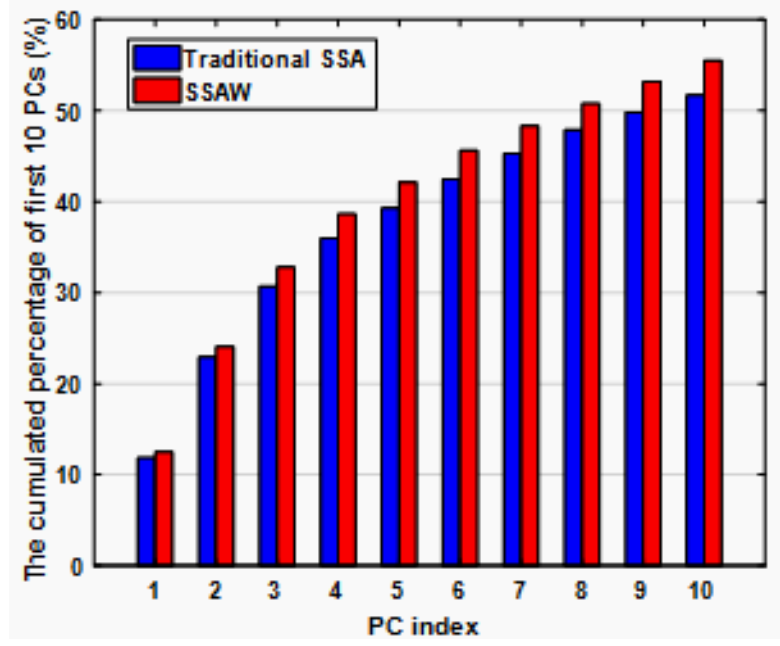

Fig. 8 The cumulated percentage of signal variance for first 10 PCs.
$55.5 \%$ of the total variance, much larger than that $(51.3 \%)$ of the original time series.

The signals, which are reconstructed from the first 10 PCs with both the SSAW and traditional SSA, are presented in Figure 9 and their differences in bottom panel. It is apparent that the differences are clearly correlated with the formal errors of the time series. If the weights of the SSAW are determined in such a way that the sum of the weights is equal to the epochs number $N$, the root mean squared error of unit weight indicates the fitting error of the SSAW. According to the definition of weight, the weight of the GMSL time series is defined as,

$p_{i}=\sigma_{0}^{2} / \alpha_{i}^{2}, \quad i=1,2, \cdots, N$

where $\sigma_{0}^{2}$ is the variance of unit weight, $\sigma_{i}$ is the formal error at $i$ th epoch. Since $\sum_{i=1}^{N} p_{i}=N$, we can solve $\sigma_{0}^{2}$ from Equation (23), that is,

$\sigma_{0}^{2}=N / \sum_{i=1}^{N}\left(1 / \alpha_{i}^{2}\right)$

Substituting (24) into (23) yields the weight as,

$p_{i}=N /\left(\alpha_{i}^{2} \sum_{k=1}^{N}\left(1 / \alpha_{k}^{2}\right)\right)$ 


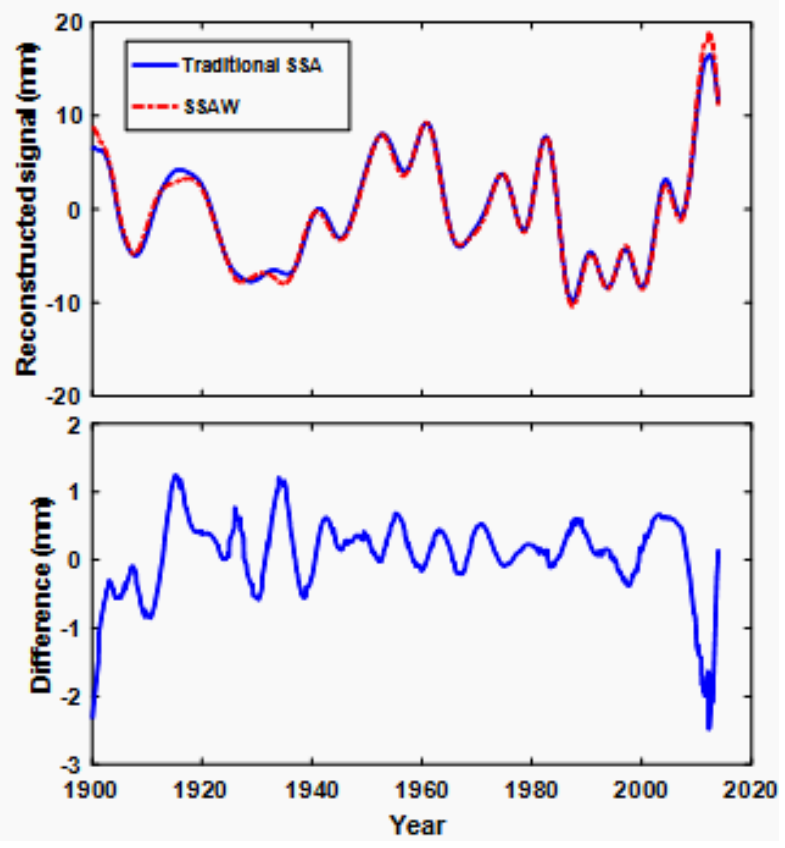

Fig. 9 Extracted signals of the traditional and SSAW (top) and their difference (bottom).

If the signals reconstructed with the SSAW at $i$ th epoch is expressed with $\hat{x}_{i}^{w}$, the fitting error of the SSAW is estimated by,

$m_{w}=\sqrt{\frac{1}{N} \sum_{i=1}^{N} p_{i}\left(x_{i}-\hat{x}_{i}^{w}\right)^{2}}$

Similarly for traditional SSA, the unit weight adopted, the fitting error is estimated by,

$$
m_{s}=\sqrt{\frac{1}{N} \sum_{i=1}^{N}\left(x_{i}-\hat{x}_{i}^{s}\right)^{2}}
$$

where $\hat{x}_{i}^{s}$ is the reconstructed signal with traditional SSA at $i$ th epoch.

The fitting errors of the GMSL time series using 10 PCs are $4.80 \mathrm{~mm}$ and $5.14 \mathrm{~mm}$ for the SSAW and traditional SSA, respectively. Compared to traditional SSA, the SSAW can reduce the fitting error about $6.61 \%$. Therefore, when the formal errors of a noisy time series are available, the SSAW is suggested to deal with the time series.

\section{SIMULATION STUDIES BASED ON THE RECONSTRUCTED GMSL SIGNALS}

To demonstrate the performance of the SSAW, we carry out simulation studies based on the real signals shown in Figure 9 extracted by traditional SSA approach. The synthetic time series are generated for 500 times by Equation (11) where noise is the normally distributed noise with zero mean and variance $\sigma_{i}^{2}$. The real $\sigma_{i}^{2}$ are the formal errors of GMSL time series shown in Figure 2. The same window size $(L=600)$ is chosen and first 10 PCs are selected to reconstruct signals. Since the real signal is known, we can compute the root mean squared error (RMSE) and mean absolute error (MAE) with the differences between the reconstructed signal and simulated real signal. They are defined as the following equations:

$$
\begin{aligned}
& \mathrm{RMSE}=\sqrt{\frac{1}{N} \sum_{i=1}^{N}\left(s_{i}-\hat{x}_{i}\right)^{2}} \\
& \mathrm{MAE}=\frac{1}{N} \sum_{i=1}^{N}\left|s_{i}-\hat{x}_{i}\right|
\end{aligned}
$$

where $\hat{x}_{i}$ denotes the reconstructed signal at $i$ th epoch, $N$ is the number of observations. The relative improvements (IMP) of RMSE and MAE of the SSAW with respect to traditional SSA is computed with,

$$
\begin{gathered}
\mathrm{IMP}_{\mathrm{RMSE}}=\frac{\mathrm{RMSE}_{\mathrm{s}}-\mathrm{RMSE}_{\mathrm{w}}}{\mathrm{RMSE} \mathrm{s}_{\mathrm{s}}} \times 100 \% \\
\mathrm{IMP}_{\mathrm{MAE}}=\frac{\mathrm{MAE}_{\mathrm{s}}-\mathrm{MAE}_{\mathrm{w}}}{\mathrm{MAE}_{\mathrm{s}}} \times 100 \%
\end{gathered}
$$

where the subscript ' $w$ ' and ' $s$ ' represent the values of the SSAW and traditional SSA, respectively.

The RMSEs and MAEs for the SSAW and traditional SSA are presented in Figure 10, where all the RMSEs and MAEs of the SSAW are smaller than those of traditional SSA. The average RMSE and

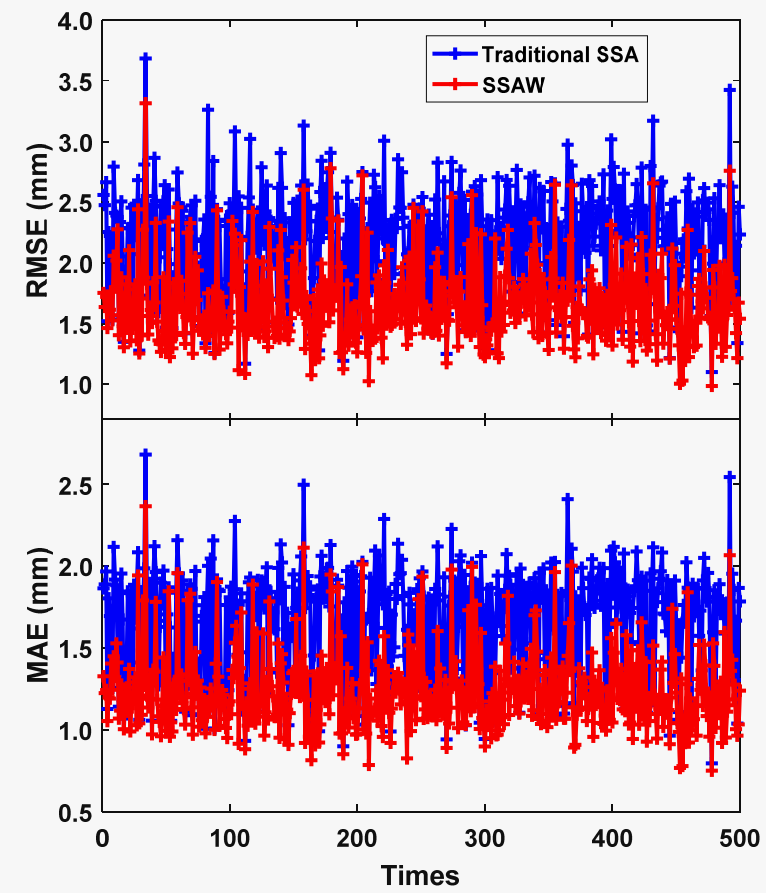

Fig. 10 RMSEs and MAEs of 500 simulations of the SSAW and traditional SSA. 
MAE are $1.67 \mathrm{~mm}$ and $1.34 \mathrm{~mm}$ for the SSAW, $2.18 \mathrm{~mm}$ and $1.66 \mathrm{~mm}$ for traditional SSA, respectively. The corresponding IMPs of RMSE and MAE are $23.85 \%$ and $19.76 \%$, respectively. Therefore, the proposed SSAW effectively extracts signals from noisy series compared to traditional SSA

\section{CONCLUSIONS}

In order to more efficiently extract signals from noisy time series, the SSAW approach is proposed by considering the formal errors of the time series. The GMSL time series are processed by using the SSAW and traditional SSA; the results demonstrate that the SSAW achieves better performance than traditional SSA in reconstructing signals. The first $10 \mathrm{PCs}$ of the SSAW and traditional SSA contain 55.5 \% and $51.7 \%$ of total variance, respectively. When the first $10 \mathrm{PCs}$ are used to reconstruct signals for fitting the original time series, the fitting error of the SSAW is $4.80 \mathrm{~mm}$, much smaller than $5.14 \mathrm{~mm}$, the fitting error of traditional SSA. Moreover, according to the reconstructed GMSL signals and formal errors of the GMSL time series, 500 simulations are carried out. The statistics show that the mean RMSE and MAE of the SSAW relative to traditional SSA are reduced from $2.18 \mathrm{~mm}$ to $1.67 \mathrm{~mm}, 1.66 \mathrm{~mm}$ to $1.34 \mathrm{~mm}$, with improvements $23.85 \%$ and $19.76 \%$, respectively. All the RMSEs and MAEs of the SSAW are smaller than those of traditional SSA. Therefore, when the formal errors of a time series are available, the SSAW approach is suggested to be applied to process this time series.

\section{ACKNOWLEDGMENTS}

This work is mainly sponsored by the National Key R\&D Program of China (2017YFA0603103) and the Natural Science Foundation of China (Projects: 41731069 and 41274035$)$. We thank two anonymous reviewers for their constructive comments.

\section{REFERENCES}

Allen, M. and Smith, L.: 1997, Optimal filtering in singular spectrum analysis. Phys. Lett. A, 234, 6, 419-428. DOI: $10.1016 / \mathrm{s} 0375-9601(97) 00559-8$

Barrios-Muriel, J., Romero F., Alonso, F. J. and Gianikellis, K.: 2016, A simple SSA-based de-noising technique to remove ECG interference in EMG signals. Biomed. Signal Proces. Control, 30, 117-126. DOI: 10.1016/j.bspc.2016.06.001

Broomhead, D. and King, G.: 1986, Extracting qualitative dynamics from experimental data. Physica D: Nonlinear Phenomena, 20, 2-3, 217-236. DOI: $10.1016 / 0167-2789(86) 90031-X$

Cadzow, J.: 1988, Signal enhancement - a composite property mapping algorithm. IEEE Trans. Speech Audio Process, 36, 1, 49-62. DOI: 10.1109/29.1488

Chambers, D., Merrifield, M. and Nerem, R.: 2012, Is there a 60-year oscillation in global mean sea level? Geophys. Res. Lett., 39, L18607. DOI: $10.1029 / 2012$ GL052885

Chen, J., Wilson, C., Chambers, D., Nerem, R. and Tapley, B.: 1998, Seasonal global water mass budget and mean sea level variations. Geophys. Res. Lett., 25, 19, 3555-3558. DOI: 10.1029/98GL02754

Chen, Q. and Sneeuw, N.: 2016, Singular spectrum analysis for modeling geodetic time series. IAG Symposia. DOI: $10.1007 / 1345 \_2016 \_248$

Church, J. and White, N.: 2011, Sea-level rise from the late 19 th to the early 21 st century. Surv. Geophys., 32, 4-5, 585-602. DOI: 10.1007/s10712-011-9119-1

Figueiredo, M., Almeida, A. and Ribeiro, B.: 2011, Wavelet decomposition and singular spectrum analysis for electrical signal denoising. Proc. IEEE Int. Conf. on Systems, Man and Cybernetics, 3329-3334. DOI: 10.1109/ICSMC.2011.6084183

Gillard, J. W. and Zhigljavsky, A.: 2016, Weighted norms in subspace-based methods for time series analysis. Numer. Linear Algebr. Appl., 23, 5, 947-967. DOI: $10.1002 /$ nla.2062

Golyandina, N.: 2010, On the choice of parameters in singular spectrum analysis and related subspace-based methods. Stat. Interface, 3, 3, 259-279.

Golyandina, N., Korobeynikov, A. and Zhigljavsky, A.: 2018, Singular spectrum analysis with R. Springer, 270 pp. DOI: $10.1007 / 978-3-662-57380-8$

Golyandina, N., Nekrutkin, V. and Zhigljavsky, A.: 2001, Analysis of time series structure: SSA and related techniques. Chapman \& Hall/CRC, Boca Raton. DOI: $10.1201 / 9781420035841$

Golyandina, N. and Zhigljavsky, A.: 2013, Singular spectrum analysis for time series. Springer, $119 \mathrm{pp}$. DOI: 10.1007/978-3-642-34913-3.

Hansen P. and Jensen, S.: 2005, Prewhitening for rankdeficient noise in subspace methods for noise reduction. IEEE Trans. Signal Process, 53, 10, 37183726. DOI: $10.1109 /$ TSP. 2005.855110

$\mathrm{Hu}$, B., Li, Q. and Smith, A.: 2009, Noise reduction of hyper-spectral data using singular spectral analysis. Int. J. Remote Sens., 30, 9, 2277-2296. DOI: 10.1109/TSP.2005.855110

Kondrashov, D. and Ghil, M.: 2006, Spatiotemporal filling of missing points in geophysical data sets. Nonlinear Proc. Geoph., 13, 2, 151-159. DOI: $10.5194 / \mathrm{npg}-13-151-2006$

Kondrashov, D., Shprits, Y. and Ghil, M.: 2010, Gap filling of solar wind data by singular spectrum analysis. Geophys. Res.Lett., 37,15, L15101, 78-82. DOI: $10.1029 / 2010$ GL044138

Kumar, K., Rajesh, R. and Tiwari, R.: 2017, Regional and residual gravity anomaly separation using the singular spectrum analysis-based low pass filtering: a case study from Nagpur, Maharashtra, India. Explor. Geophys., 49, 3, 398-408. DOI: 10.1071/EG16115

Li, W., Shen, Y. and Li, B.: 2015, Weighted spatiotemporal filtering using principal component analysis for analyzing regional GNSS position time series. Acta Geod. Geophys., 50, 4, 419-436. DOI: $10.1007 / \mathrm{s} 40328-015-0100-1$

Ma, J., Li, Z. and Wang, B.: 2011, Application of singular spectrum analysis to the noise reduction of intrusion detection alarms. J. Computers, 6, 8, 1715-1722. DOI: $10.4304 /$ jcp.6.8.1715-1722

Nerem, R.: 1995, Measuring global mean sea level variations using TOPEX/POSEIDON altimeter data. J. Geophys. Res. Atmos., 100, C12, 25135-25151. DOI: 10.1029/954JC02303

Oropeza, V. and Sacchi, M.: 2011, Simultaneous seismic data denoising and reconstruction via multichannel singular spectrum analysis. Geophysics, 76, 3, 25-32. DOI: $10.1190 / 1.3552706$ 
Rajesh, R. and Tiwari, R.: 2014, Windowed SSA (Singular Spectral Analysis) for geophysical time series analysis. J. Geological Resource and Engineering, 1, 3, 167-173. DOI: $10.17265 / 2328-2193 / 2014.03 .004$

Rangelova, E., Sideris, M. and Kim, J.: 2012, On the capabilities of the multi-channel singular spectrum method for extracting the main periodic and nonperiodic variability from weekly GRACE data. J. Geodyn., 54, 64-78. DOI: 10.1016/j.jog.2011.10.006

Robertson, A. and Mechoso, C.: 1998, Interannual and decadal cycles in river flows of southeastern South America. J. Climate, 11. 10, 2570-2581.

DOI: $10.1175 / 1520$ 0442(1998)011<2570:IADCIR>2.0.CO;2

Schoellhamer, D.: 2001, Singular spectrum analysis for time series with missing data. Geophys. Res. Lett., 28, 16, 3187-3190. DOI: 10.1029/2000GL012698

Shen, Y., Li, W., Xu, G. and Li, B.: 2014, Spatiotemporal filtering of regional GNSS network's position time series with missing data using principle component analysis. J. Geodesy, 88, 1, 1-12.

DOI: 10.1007/s00190-013-0663-y

Shen, Y., Peng, F. and Li, B.: 2015, Improved singular spectrum analysis for time series with missing data. Nonlinear Proc. Geoph., 22, 4, 371-376. DOI: 10.5194/npg-22-371-2015

Sivapragasam, C., Liong, S. and Pasha, M.: 2001, Rainfall and runoff forecasting with SSA-SVM approach. J. Hydroinform., 3, 3, 141-152.

Tiwari, R. and Rajesh, R.: 2014, Factorized Hankel optimal singular spectral approach for erratic and noisy seismic signal denoising. J. Appl. Geophys., 111, 95101. DOI: $10.1016 /$ j.jappgeo.2014.09.019

Vautard, R. and Ghil, M.: 1989, Singular spectrum analysis in nonlinear dynamics with applications to paleoclimatic time series. Physica D, 35, 3, 395-424. DOI: $10.1016 / 0167-2789(89) 90077-8$
Vautard, R., Yiou, P. and Ghil, M.: 1992, Singular-spectrum analysis: A toolkit for short, noisy chaotic signals. Physica D, 58, 1-4, 95-126. DOI: 10.1016/0167-2789(92)90103-T

Vitanov, N., Sakai, K. and Dimitrova, Z.: 2008, SSA, PCA, TDPSC, ACFA: Useful combination of methods for analysis of short and nonstationary time series. Chaos Soliton Fract., 37, 1, 187-202. DOI: $10.1016 /$ j.chaos.2006.08.043

Watson, P.J.: 2016, Identifying the best performing time series analytics for sea level research. In book Time Series Analysis and Forecasting. Springer, 261-278.

Wu, C. and Chau, K.: 2011, Rainfall-runoff modeling using artificial neural network coupled with singular spectrum analysis. J. Hydrol., 399, 3-4, 394-409.

Zotov, L., Bizouard, C. and Shum, C.: 2016, A possible interrelation between Earth rotation and climate variability on a decadal time-scale. J. Geodesy Geodyn., 7, 3, 216-222.

DOI: $10.1016 /$ j.geog.2016.05.005

Zotov, L. and Shum, C.: 2010, Multichannel singular spectrum analysis of the gravity field data from grace satellites. AIP Conference Proceedings, 1206, 1, 473 479. DOI: $10.1063 / 1.3292557$

Zotov, L., Sidorenkov, N., Bizouard, C., Shum, C. and Shen, W.: 2017, Multichannel singular spectrum analysis of the axial atmospheric angular momentum. J. Geodesy Geodyn., 8, 6, 433-442. DOI: $10.1016 /$ j.geog.2017.02.010

Zvonarev, N. and Golyandina, N.: 2017, Iterative algorithms for weighted and unweighted finite-rank time-series approximations. Stat. Interface, 10, 1, 5-18. DOI: 10.4310/SII.2017.v10.n1.a1 